\title{
DIFFERENTIAL ALGEBRAIC DEPENDENCE AND NOVIKOV DEPENDENCE
}

\author{
Bibinur Duisengaliyeva $]^{1}$ Ualbai Umirbaev
}

\begin{abstract}
We define an analogue of the Fox derivatives for differential polynomial algebras and give a criterion for differential algebraic dependence of a finite system of elements. In particular, we prove that differential algebraic dependence of a finite set of elements of a differential polynomial algebra over a constructive differential field $k$ of characteristic zero is algorithmically recognizable. Using a representation of free Novikov algebras by differential polynomials we also give a criterion of Novikov dependence of a finite system of elements of free Novikov algebras.
\end{abstract}

Mathematics Subject Classification (2010): Primary 12H05, 17D25; Secondary 12L05, 13P10, 17A50.

Key words: Differential polynomial algebra, differential algebraic dependence, free Novikov algebra, Novikov dependence.

\section{INTRODUCTION}

Algebraic dependence of a finite set of elements of a polynomial algebra $k\left[x_{1}, x_{2}, \ldots, x_{n}\right]$ over a constructive field $k$ is algorithmically recognizable [17] by the methods of Gröbner bases [2]. The concept of algebraic dependence is easily generalized to arbitrary varieties of algebras. For example, a system of elements $f_{1}, f_{2}, \ldots, f_{m}$ of an arbitrary associative algebra $A$ is called associatively independent if the subalgebra generated by these elements is a free associative algebra with free generators $f_{1}, f_{2}, \ldots, f_{m}$. Otherwise the elements $f_{1}, f_{2}, \ldots, f_{m}$ are called associatively dependent.

By the Nielsen-Schreier Theorem the subgroups of free groups are free [12] and by the Shirshov-Witt Theorem the subalgebras of free Lie algebras are free [19, 23]. These results easily imply that the dependence of a finite system of elements of free groups and free Lie algebras are algorithmically recognizable. Associative dependence of a finite system of elements of free associative algebras [20] is algorithmically unrecognizable.

This paper is devoted to the study of differential algebraic dependence and Novikov dependence. The basic concepts of differential algebras can be found in [9, 16]. Let $\Delta=\left\{\delta_{1}, \ldots, \delta_{m}\right\}$ be a basic set of derivation operators. If $\Delta$ contains only one element, then differential algebras are called ordinary, and if $\Delta$ contains at least two elements, then they are called partial.

\footnotetext{
1 L.N. Gumilyov Eurasian National University, Astana, 010008, Kazakhstan, e-mail: bibinur.88@mail.ru

2 Wayne State University, Detroit, MI 48202, USA, and Institute of Mathematics and Modeling, Almaty, Kazakhstan, e-mail: umirbaev@math.wayne.edu

The project is partially supported by the grant AP05133009 of MES RK
} 
The differential polynomial algebra $k\left\{x_{1}, x_{2}, \ldots, x_{n}\right\}$ in the variables $x_{1}, x_{2}, \ldots, x_{n}$ over a differential field $k$ is the closest generalization of the polynomial algebra $k\left[x_{1}, x_{2}, \ldots, x_{n}\right]$. However, the structures of the subalgebras and ideals of these algebras are very different. It is well known that the ideal membership problem (see, for example [2]) and the subalgebra membership problem [17, 15] for polynomial algebras are algorithmically decidable. At the same time the ideal membership problem and the subalgebra membership problem for partial differential polynomial algebras is algorithmically undecidable [22]. These questions remain open for ordinary differential polynomial algebras [11.

A set of elements $f_{1}, f_{2}, \ldots, f_{m}$ of the polynomial algebra $k\left[x_{1}, \ldots, x_{n}\right]$ over a field $k$ of characteristic zero is algebraically dependent if and only if the rank of the Jacobian matrix

$$
J\left(f_{1}, f_{2}, \ldots, f_{m}\right)=\left[\frac{\partial f_{i}}{\partial x_{j}}\right]_{1 \leq i \leq m, 1 \leq j \leq n}
$$

is less than $m$ (see, for example [18]). In this paper we formulate and prove an analogue of this result for the differential polynomial algebra $k\left\{x_{1}, x_{2}, \ldots, x_{n}\right\}$ over a differential field $k$ of characteristic zero in the terms of Fox derivatives (see, for example [21]). This result allows us to prove that the differential algebraic dependence of a finite system of elements of differential polynomial algebras over a constructive field of characteristic zero is algorithmically recognizable.

I.M. Gel'fand and I.Ya. Dorfman [7] noticed that any differential algebra with derivation $\delta$ with respect to the multiplication $a \circ b=a(\delta b)$ becomes a Novikov algebra. In [5] free Novikov algebras are represented by ordinary differential polynomial algebras via the multiplication $a \circ b=a(\delta b)$. Using this representation of free Novikov algebras, we show that a finite system of elements of a free Novikov algebra is Novikov dependent if and only if it is differentially algebraically dependent. In particular, the Novikov dependence of a finite system of elements of free Novikov algebras is also algorithmically recognizable. This also implies that any $n+1$ elements of a free Novikov algebra of rank $n$ are Novikov dependent.

It is well known [6] that any two algebraically dependent elements of a polynomial algebra are polynomials in one variable. Any two associatively dependent elements of a free associative algebra are also polynomials in one variable [1. L. Makar-Limanov and I. Shestakov [13] (see also [21, 14]) proved an analogue of this result for free Poisson algebras over fields of characteristic zero. The question on the validity of similar results for differential polynomial algebras and for free Novikov algebras remains open.

The rest of the paper is organized as follows. In Section 2 we give some elementary terminology on differential polynomial algebras. The definition of the Fox derivatives for differential polynomial algebras is given in Section 3. Section 3 is devoted to the study of differential algebraic dependence in differential polynomial algebras. In Section 4 we recall a representation of free Novikov algebras in differential polynomial algebras. Using this reperesentation, in Section 5, we describe Novikov dependence of elements in free Novikov algebras.

\section{Differential polynomial algebras}


Let $\Delta=\left\{\delta_{1}, \ldots, \delta_{m}\right\}$ be a basic set of derivation operators. A commutative $\operatorname{ring} R$ with identity is called a differential ring or $\Delta$-ring if all elements of $\Delta$ act on $R$ as a commuting set of derivations, i.e., the derivations $\delta_{i}: R \rightarrow R$ are defined for all $i$ and $\delta_{i} \delta_{j}=\delta_{j} \delta_{i}$ for all $i, j$.

Let $\Theta$ be the free commutative monoid on the set $\Delta=\left\{\delta_{1}, \ldots, \delta_{m}\right\}$ of derivation operators. The elements

$$
\theta=\delta_{1}^{i_{1}} \ldots \delta_{m}^{i_{m}}
$$

of the monoid $\Theta$ are called derivative operators. The order of $\theta$ is defined as $|\theta|=$ $i_{1}+\ldots+i_{m}$. Let $\gamma(\theta)=\left(i_{1}, \ldots, i_{m}\right) \in \mathbb{Z}_{+}^{m}$, where $\mathbb{Z}_{+}$is the set of all nonnegative integers.

Let $R$ be a differential ring. Denote by $R[\Delta]$ the free left $R$-module with a basis $\Theta$. Every element $u \in R[\Delta]$ can be uniquely written in the form

$$
u=\sum_{\theta \in \Theta} r_{\theta} \theta
$$

with a finite number of nonzero $r_{\theta} \in R$. We turn $R[\Delta]$ to a ring by

$$
\delta_{i} r=r \delta_{i}+\delta_{i}(r)
$$

for all $i$ and $r \in R$. It is well known [10] that these relations uniquely define a structure of a ring on $R[\Delta]$. The ring $R[\Delta]$ is called the ring of differential operators with coefficients in $R$. Every left module over $R[\Delta]$ is called a differential module over $R$. In particular, $R$ is a left $R[\Delta]$ and every $I \subseteq R$ is a differential ideal of $R$ if and only if $I$ is an $R[\Delta]$ submodule of $R$. This means that $R[\Delta]$ is the universal enveloping ring of $R$ in the usual ring-theoretic terminology.

Let $x^{\Theta}=\left\{x^{\theta} \mid \theta \in \Theta\right\}$ be a set of symbols enumerated by the elements of $\Theta$. Consider the polynomial algebra $R\left[x^{\Theta}\right]$ over $R$ generated by the set of (polynomially) independent variables $x^{\Theta}$. It is easy to check that the derivations $\delta_{i}$ can be uniquely extended to a derivation of $R\left[x^{\Theta}\right]$ by $\delta_{i}\left(x^{\theta}\right)=x^{\delta_{i} \theta}$. Denote this differential ring by $R\{x\}$; it is called the ring of differential polynomials in $x$ over $R$.

By adjoining more variables, we can obtain the differential ring $R\left\{x_{1}, x_{2}, \ldots, x_{n}\right\}$ of the differential polynomials in $x_{1}, x_{2}, \ldots, x_{n}$ over $R$. Let $M$ be the free commutative monoid generated by all elements $x_{i}^{\theta}$, where $1 \leq i \leq n$ and $\theta \in \Theta$. The elements of $M$ are called differential monomials of $R\left\{x_{1}, x_{2}, \ldots, x_{n}\right\}$. Every element $a \in R\left\{x_{1}, x_{2}, \ldots, x_{n}\right\}$ can be uniquely written in the form

$$
a=\sum_{m \in M} r_{m} m
$$

with a finite number of nonzero $r_{m} \in R$.

If $R$ is a domain then the field of fractions $R\left\langle x_{1}, x_{2}, \ldots, x_{n}\right\rangle$ of $R\left\{x_{1}, x_{2}, \ldots, x_{n}\right\}$ becomes a differential field since every $\delta_{i}$ can be uniquely extended to $R\left\langle x_{1}, x_{2}, \ldots, x_{n}\right\rangle$. This field is called the field of rational differential functions in the variables $x_{1}, x_{2}, \ldots, x_{n}$ over $R$.

\section{Fox DERIVATIVES}

Let $k$ be an arbitrary differential field of characteristic zero and let $A=k\left\{x_{1}, x_{2}, \ldots, x_{n}\right\}$ be the differential polynomial algebra over $k$ in the variables $x_{1}, x_{2}, \ldots, x_{n}$. Let $B=$ 
$k\left\langle x_{1}, x_{2}, \ldots, x_{n}\right\rangle$. We are going to define analogues of the Fox derivatives for $A$ and $B$. Let $C=k\left\langle x_{1}, x_{2}, \ldots, x_{n}, y_{1}, y_{2}, \ldots, y_{n}\right\rangle$ be the differential field with additional variables $y_{1}, y_{2}, \ldots, y_{n}$ and $B \subset C$. Let $\lambda$ be the derivation of the differential field $C$ defined by $x_{i} \mapsto y_{i}$ and $y_{i} \mapsto 0$ for all $i$. Put

$$
\Omega_{B}=B[\Delta] y_{1} \oplus B[\Delta] y_{2} \oplus \ldots \oplus B[\Delta] y_{n}
$$

Notice that if $f \in B$ then $\lambda(f) \in \Omega_{B}$. Denote by

$$
D: B \longrightarrow \Omega_{B}
$$

the restriction of $\lambda$. Then $D$ is a derivation of $B$ with coefficients in the free left $B[\Delta]$ module $\Omega_{B}$. Obviously, $D$ is the universal derivation of $B$ (see, for example [21]). For any $f \in B$ there are unique elements $a_{1}, \ldots, a_{n} \in B[\Delta]$ such that

$$
D(f)=a_{1} y_{1}+\ldots+a_{n} y_{n} .
$$

Let $a_{i}=d_{x_{i}}(f)$ for all $i$. This defines the derivations

$$
d_{x_{i}}: B \longrightarrow B[\Delta]
$$

such that $d_{x_{i}}\left(x_{j}\right)=\delta_{i j}$, where $\delta_{i j}$ is the Kronecker delta. These derivations are analogues of Fox derivatives. Let

$$
d(f)=\left(d_{x_{1}}(f), \ldots, d_{x_{n}}(f)\right), \quad f \in B .
$$

If $f_{1}, \ldots, f_{m} \in B$ then the matrix

$$
J\left(f_{1}, \ldots, f_{m}\right)=\left[d_{x_{j}}\left(f_{i}\right)\right]_{1 \leq i \leq m, 1 \leq j \leq n}
$$

is called the Jacobian matrix of $f_{1}, \ldots, f_{m}$. The row vectors $d\left(f_{1}\right), \ldots, d\left(f_{m}\right)$ are rows of $J\left(f_{1}, \ldots, f_{m}\right)$. The Chain Rule can be written in the form

$$
d\left(f\left(g_{1}, \ldots, g_{n}\right)\right)=d(f) J\left(g_{1}, \ldots, g_{n}\right) .
$$

Notice that if $f \in A$ then $d_{x_{i}}(f) \in A[\Delta]$. Let $\varphi$ be an endomorphism of $A$ that sends $x_{i}$ to $f_{i}$. Then

$$
J(\varphi)=J\left(f_{1}, \ldots, f_{n}\right)
$$

is the Jacobian matrix of $\varphi$. The Chain Rule implies that $J(\varphi)$ is invertible over $A[\Delta]$ if $\varphi$ is an automorphism. The Jacobian Conjecture for differential polynomial algebras can be formulated as follows: Is any endomorphism $\varphi$ of a differential polynomial algebra $A$ an automorphism if $J(\varphi)$ is invertible over $A[\Delta]$ ? The study of the Jacobian Conjecture for differential polynomial algebras is interesting first of all in the perspective of constructing a counter example. Partial differential polynomial algebras have wild automorphisms even in two variables [3].

\section{Differential Algebraic DePendence}

Elements $f_{1}, \ldots, f_{m}$ of $B=k\left\langle x_{1}, x_{2}, \ldots, x_{n}\right\rangle$ are called differentially algebraically dependent if there exists a nonzero element $g$ of a differential polynomial algebra $k\left\{y_{1}, \ldots, y_{m}\right\}$ in the variables $y_{1}, \ldots, y_{m}$ such that $g\left(f_{1}, \ldots, f_{m}\right)=0$. Obviously, the elements $f_{1}, \ldots, f_{m}$ are differentially algebraically dependent if and only if the elements $f_{1}^{\theta}, \ldots, f_{m}^{\theta}$, where $\theta \in \Theta$, are algebraically dependent. The set of elements $f_{1}^{\theta}, \ldots, f_{m}^{\theta}$, where $\theta \in \Theta$, is 
infinite and we cannot check algebraic dependence of this set by using the algorithms for polynomial algebras.

Theorem 1. Elements $f_{1}, \ldots, f_{m}$ of $B=k\left\langle x_{1}, x_{2}, \ldots, x_{n}\right\rangle$ are differentially algebraically dependent if and only if $d\left(f_{1}\right), \ldots, d\left(f_{m}\right)$ are left dependent over $B[\Delta]$.

Proof. Elements $f_{1}, \ldots, f_{m}$ are differentially algebraically dependent if and only if the elements $f_{1}^{\theta}, \ldots, f_{m}^{\theta}$, where $\theta \in \Theta$, are algebraically dependent. An infinite set of elements is algebraically dependent if and only if it contains a finite algebraically dependent subset. Let $\theta_{1,1}, \ldots, \theta_{1, s_{1}}, \theta_{2,1}, \ldots, \theta_{m, s_{m}}$ be elements of $\Theta$ such that $\theta_{i, j} \neq \theta_{i, t}$ if $j \neq t$ and the elements

$$
f_{1}^{\theta_{1,1}}, \ldots, f_{1}^{\theta_{1, s_{1}}}, f_{2}^{\theta_{2,1}}, \ldots, f_{m}^{\theta_{m, s_{m}}}
$$

are algebraically dependent.

Let $e_{1}, \ldots, e_{r}$ be the minimal subset of $X^{\Theta}$ such that the set of elements (11) is contained in $k\left[e_{1}, \ldots, e_{r}\right]$. For any $f \in k\left[e_{1}, \ldots, e_{r}\right]$ let

$$
\partial(f)=\left(\frac{\partial f}{\partial e_{1}}, \ldots, \frac{\partial f}{\partial e_{r}}\right)
$$

where $\frac{\partial}{\partial e_{i}}$ is the usual partial derivation of the polynomial algebras. The elements (10) are algebraically dependent [18] if and only if the elements

$$
\partial\left(f_{1}^{\theta_{1,1}}\right), \ldots, \partial\left(f_{1}^{\theta_{1, s_{1}}}\right), \partial\left(f_{2}^{\theta_{2,1}}\right), \ldots, \partial\left(f_{m}^{\theta_{m, s_{m}}}\right)
$$

are left dependent over $k\left[e_{1}, \ldots, e_{r}\right]$. Suppose that

$$
\sum_{i, j} a_{i j} \partial\left(f_{i}^{\theta_{i, j}}\right)=0
$$

where $a_{i j} \in k\left[e_{1}, \ldots, e_{r}\right]$ and at least one $a_{i j}$ is nonzero.

Notice that for any $f \in k\left[e_{1}, \ldots, e_{r}\right]$ we have

$$
d(f)=\partial(f) J\left(e_{1}, \ldots, e_{r}\right) .
$$

Consequently, multiplying (2) by $J\left(e_{1}, \ldots, e_{r}\right)$ from the right hand side, we get

$$
\sum_{i, j} a_{i j} d\left(f_{i}^{\theta_{i, j}}\right)=0
$$

Since $d$ commutes with the elements of $\Delta$, this equality can be written as

$$
\sum_{i, j} a_{i j} \theta_{i, j} d\left(f_{i}\right)=\sum_{i}\left(\sum_{j} a_{i j} \theta_{i, j}\right) d\left(f_{i}\right)=\sum_{i} b_{i} d\left(f_{i}\right)=0
$$

where $b_{i}=\sum_{j} a_{i j} \theta_{i, j}$. If $a_{i j} \neq 0$, then $b_{i} \neq 0$, since $\theta_{i, j} \neq \theta_{i, t}$ if $j \neq t$ and $d\left(f_{1}\right), \ldots, d\left(f_{m}\right)$ are left dependent over $B[\Delta]$. Conversely, every dependence of this form can be uniquely written in the form (2).

Lemma 1. Let $k$ be a constructive differential field. Then for any two nonzero elements $a$ and $b$ of the algebra $B[\Delta]$ there can be effectively found two elements $c, d \in B[\Delta]$ such that $c a=d b \neq 0$. 
Proof. It is well known that if $R$ is a left Noetherian domain with a derivation $\delta$ then the skew polynomial ring $R[x, \delta]$ is again a left Noetherian domain [8].

Let $B_{i}$ be the subalgebra of $B[\Delta]$ generated by $B$ and $\delta_{1}, \ldots, \delta_{i}$, where $0 \leq i \leq m$. In particular, $B_{0}=B$ and $B_{m}=B[\Delta]$. Notice that $\delta_{i+1}$ can be considered as a derivation of $B_{i}$ with trivial zero action on $\delta_{1}, \ldots, \delta_{i}$. Then $B_{i+1} \cong B_{i}\left[\delta_{i+1}\right]$ is a skew polynomial ring over $B_{i}$. Consequently, $B[\Delta]$ is a left Noetherian domain.

It is also well known that every left Noetherian domain satisfies the Ore condition, that is, for any nonzero elements $a, b$ there exist $c, d$ such that $c a=d b \neq 0$. This proves the existence of elements $c, d$ in the formulation of the lemma. Notice that $a, b$ are left dependent if and only if there exists a nonnegative integer $s$ such that the set of elements $a^{\theta}, b^{\theta}$, where $\theta \in \Theta$ and $|\theta| \leq s$, is left dependent over $B$. The latter is algorithmically recognizable since $B$ is a constructive field. Starting from 0 , we can find the minimal $s$ satisfying this condition and a nontrivial equality

$$
\sum_{|\theta| \leq s} c_{\theta} a^{\theta}=\sum_{|\theta| \leq s} d_{\theta} b^{\theta},
$$

where $c_{\theta}, d_{\theta} \in B$. Let

$$
c=\sum_{|\theta| \leq s} c_{\theta} \theta, \quad d=\sum_{|\theta| \leq s} d_{\theta} \theta
$$

Then (3) means that $c a=d b$. The nontriviality of (3) implies that one of the elements $c$ and $d$ is nonzero. Then $c a=d b \neq 0$ since $B[\Delta]$ is a domain.

Lemma 2. Let $k$ be a constructive differential field. Then the left dependency of a finite system of elements of a free left $B[\Delta]$-module $B[\Delta]^{s}$ is algorithmically recognizable.

Proof. Let $Q$ be the left classical quotient ring of $B[\Delta]$. Recall that every element of $Q$ can be represented in the form $a^{-1} b$, where $a, b \in B[\Delta]$. We want to show that $Q$ is a constructive skew field. In fact, $\left(a^{-1} b\right)^{-1}=b^{-1} a$ and

$$
a^{-1} b c^{-1} d=a^{-1} c_{1}^{-1} b_{1} d=\left(c_{1} a\right)^{-1}\left(b_{1} d\right),
$$

where $b_{1}, c_{1} \in B[\Delta]$ satisfying the condition $b c^{-1}=c_{1}^{-1} b_{1}$ can be found effectively by Lemma 1. Moreover,

$$
a^{-1} b+c^{-1} d=t^{-1}\left(r_{1} b+r_{2} d\right),
$$

where $t, r_{1}, r_{2} \in B[\Delta]$ satisfying condition $t=r_{1} a=r_{2} b$ can also be effectively found by Lemma 1 .

Since $B[\Delta]$ is an Ore ring, it follows that a finite system of elements of the free left $B[\Delta]$-module $B[\Delta]^{s}$ is left dependent if and only if they are left dependent elements of the free $Q$-module $Q^{s}$. Using the usual triangulation, we can recognize the left dependency of elements of a finite dimensional vector space over a constructive skew field $Q$.

Theorem 2. Let $k$ be a constructive differential field. Then the differential algebraic dependency of a finite system of elements of a free differential field of rational functions over $k$ is algorithmically recognizable. 
Proof. By Theorem 1, any elements $f_{1}, \ldots, f_{m}$ of $B=k\left\langle x_{1}, x_{2}, \ldots, x_{n}\right\rangle$ are differentially algebraically dependent if and only if $d\left(f_{1}\right), \ldots, d\left(f_{m}\right)$ are left dependent over $B[\Delta]$. The latter is algorithmically recognizable by Lemma 2 ,

\section{A Representation of Free Novikov ALGEBras}

A nonassociative algebra $A=(A, \circ)$ is called a (left) Novikov algebra if $A$ satisfies the following identities:

$$
\begin{aligned}
(a \circ b) \circ c-a \circ(b \circ c) & =(b \circ a) \circ c-b \circ(a \circ c), \\
(a \circ b) \circ c & =(a \circ c) \circ b,
\end{aligned}
$$

for any $a, b, c \in A$.

Let $k\left\{x_{1}, x_{2}, \ldots, x_{n}\right\}$ be the differential polynomial algebra over a field $k$ of a characteristic 0 in the variables $x_{1}, x_{2}, \ldots, x_{n}$ with one derivation $\delta$. For convenience we denote the derivatives $a^{\delta}, a^{\delta^{2}}, a^{\delta^{s}}$ by $a^{\prime}, a^{\prime \prime}, a^{(s)}$, respectively. Put $X=\left\{x_{1}, x_{2}, \ldots, x_{n}\right\}$ and by $X^{\delta}$ we denote the set of all symbols of the form $x_{i}^{(r)}$, where $1 \leq i \leq n, r \in \mathbb{Z}_{+}$. For any $x_{i}^{(r)}, x_{j}^{(s)} \in X^{\delta}$ we assume that $x_{i}^{(r)}>x_{j}^{(s)}$ if $i>j$ or $i=j, r>s$. The set $M$ of all differential monomials of the form

$$
u=x_{i_{1}}^{\left(s_{1}\right)} x_{i_{2}}^{\left(s_{2}\right)} \ldots x_{i_{t}}^{\left(s_{t}\right)}
$$

where $t \geq 0, x_{i_{j}}^{\left(s_{j}\right)} \in X^{\delta}$ for all $1 \leq j \leq t$ and $x_{i_{1}}^{\left(s_{1}\right)} \geq x_{i_{2}}^{\left(s_{2}\right)} \geq \ldots \geq x_{i_{t}}^{\left(s_{t}\right)}$, provides a linear basis for the algebra $k\left\{x_{1}, x_{2}, \ldots, x_{n}\right\}$.

For any $x_{i}^{(r)} \in X^{\delta}$ let

$$
\operatorname{deg}\left(x_{i}^{(r)}\right)=1, \quad d\left(x_{i}^{(r)}\right)=r
$$

where $1 \leq i \leq n$. If $u=a_{1} \ldots a_{s} \in M$, where $a_{1}, \ldots, a_{s} \in X^{\delta}$, then let

$$
\operatorname{deg}(u)=\operatorname{deg}\left(a_{1}\right)+\ldots+\operatorname{deg}\left(a_{s}\right), \quad d(u)=d\left(a_{1}\right)+\ldots+d\left(a_{s}\right),
$$

i.e., $\operatorname{deg}(u)$ is the degree of $u$ with respect to the variables $x_{1}, \ldots, x_{n}$ and $d(u)$ is the degree of $u$ with respect to $\delta$. Denote by $\operatorname{deg}_{x_{i}}(u)$ the degree of the monomial $u$ with respect to $x_{i}(1 \leq i \leq n)$.

Let $\rho(u)=\operatorname{deg}(u)-d(u)$ for any differential monomial $u$. It is easy to check that

$$
\operatorname{deg}(f g)=\operatorname{deg}(f)+\operatorname{deg}(g), \quad d(f g)=d(f)+d(g), \quad \rho(f g)=\rho(f)+\rho(g),
$$

and

$$
\operatorname{deg}\left(f^{(r)}\right)=\operatorname{deg}(f), \quad d\left(f^{(r)}\right)=d(f)+r, \quad \rho\left(f^{(r)}\right)=\rho(f)-r,
$$

for all $f, g \in k\left\{x_{1}, x_{2}, \ldots, x_{n}\right\}$.

The degree function $\rho$ defines a grading

$$
C=\bigoplus_{i \in \mathbb{Z}} C_{i}
$$

of algebra $C=k\left\{x_{1}, x_{2}, \ldots, x_{n}\right\}$, where $C_{i}$ is the $k$-span of differential monomials $u_{i}$ such that $\rho\left(u_{i}\right)=i$. Every non-zero element $c \in C$ can be uniquely written in the form

$$
c=c_{i_{1}}+c_{i_{2}}+\ldots+c_{i_{s}}, \quad \underset{7}{i_{1}}<\underset{i_{2}}{i_{7}}<\ldots<i_{s}, \quad 0 \neq c_{i_{j}} \in C_{i_{j}} .
$$


The element $c_{i_{s}}$ is called the highest homogeneous part of $c$ with respect to the degree function $\rho$ and will be denoted by $\bar{c}$.

Corollary 1. If $f, g \in k\left\{x_{1}, \ldots, x_{n}\right\}$ are homogeneous elements with respect to degree function $\rho$, then $f g$ and $f^{(r)}$ are also homogeneous with respect to $\rho$.

On the differential polynomial algebra $k\left\{x_{1}, \ldots, x_{n}\right\}$ we introduce a new operation $\circ$ by assuming

$$
f \circ g=f g^{\prime}, \quad f, g \in k\left\{x_{1}, x_{2}, \ldots, x_{n}\right\} .
$$

Then $k\left\{x_{1}, x_{2}, \ldots, x_{n}\right\}$ with the new operation $\circ$ becomes a Novikov algebra. Denote by $N_{0}\left\langle x_{1}, x_{2}, \ldots, x_{n}\right\rangle$ the subalgebra of this algebra generated by the elements $x_{1}, x_{2}, \ldots, x_{n}$. It is proved in [5] that $N_{0}\left\langle x_{1}, x_{2}, \ldots, x_{n}\right\rangle$ is a free Novikov algebra in the variables $x_{1}, x_{2}, \ldots, x_{n}$ without identity.

The structure of the space $N_{0}\left\langle x_{1}, x_{2}, \ldots, x_{n}\right\rangle$ can be described as follows.

Proposition 1. [4] The set of all differential monomials $u \in M$ with the condition $\rho(u)=$ 1 is a basis of the free Novikov algebra $N_{0}\left\langle x_{1}, \ldots, x_{n}\right\rangle$.

Denote by $N\left\langle x_{1}, \ldots, x_{n}\right\rangle=k \oplus N_{0}\left\langle x_{1}, \ldots, x_{n}\right\rangle=N_{0}\left\langle x_{1}, \ldots, x_{n}\right\rangle^{\#}$ the algebra obtained from $N_{0}\left\langle x_{1}, \ldots, x_{n}\right\rangle$ by formally joining the identity. Then $N\left\langle x_{1}, \ldots, x_{n}\right\rangle$ is the free Novikov algebra over $k$ in the variables $x_{1}, \ldots, x_{n}$ with identity. This representation of the free Novikov algebra $N\left\langle x_{1}, \ldots, x_{n}\right\rangle$ will be used below.

\section{Novikov DePendencE}

As in the preceeding section, let $k\left\{x_{1}, x_{2}, \ldots, x_{n}\right\}$ be the differential polynomial algebra over a field $k$ of a characteristic 0 in the variables $x_{1}, x_{2}, \ldots, x_{n}$ with one derivation $\delta$.

Lemma 3. Let $f_{1}, f_{2}, \ldots, f_{p} \in k\left\{x_{1}, \ldots, x_{n}\right\}$ be homogeneous elements with respect to the degree function $\rho$ and $\rho\left(f_{i}\right)=1$ for all $1 \leq i \leq p$. If $u \in k\left\{z_{1}, \ldots, z_{p}\right\}$ is a monomial such that $\rho(u)=s$, then the element $u\left(f_{1}, f_{2}, \ldots, f_{p}\right)$ is also homogeneous with respect to the degree function $\rho$ and

$$
\rho\left(u\left(f_{1}, f_{2}, \ldots, f_{p}\right)\right)=s .
$$

Proof. If $u=z_{i}^{(r)}$, then $\rho(u)=1-r=s$. By Corollary 1$] u\left(f_{i}\right)=f_{i}^{(r)}$ is homogeneous with respect to $\rho$ and (5) gives that

$$
\rho\left(u\left(f_{i}\right)\right)=\rho\left(f_{i}^{(r)}\right)=\rho\left(f_{i}\right)-r=1-r=s .
$$

Let $u=v w$, where $\operatorname{deg}(v), \operatorname{deg}(w)<\operatorname{deg}(u)$. Conducting induction on $\operatorname{deg}(u)$, we can assume that $v\left(f_{1}, f_{2}, \ldots, f_{p}\right)$ and $w\left(f_{1}, f_{2}, \ldots, f_{p}\right)$ are homogeneous with respect to $\rho$ and $\rho\left(v\left(f_{1}, f_{2}, \ldots, f_{p}\right)\right)=\rho(v), \rho\left(w\left(f_{1}, f_{2}, \ldots, f_{p}\right)\right)=\rho(w)$. From (4) we get

$$
\begin{array}{r}
\rho\left(u\left(f_{1}, f_{2}, \ldots, f_{p}\right)\right)=\rho\left(v\left(f_{1}, f_{2}, \ldots, f_{p}\right) w\left(f_{1}, f_{2}, \ldots, f_{p}\right)\right) \\
=\rho\left(v\left(f_{1}, f_{2}, \ldots, f_{p}\right)\right)+\rho\left(w\left(f_{1}, f_{2}, \ldots, f_{p}\right)\right)=\rho(v)+\rho(w)=s .
\end{array}
$$

The elements $f_{1}, f_{2}, \ldots f_{p}$ of the Novikov algebra $N\left\langle x_{1}, x_{2}, \ldots, x_{n}\right\rangle$ are called Novikov dependent if there exists a nonzero element $h\left(z_{1}, z_{2}, \ldots, z_{p}\right) \in N\left\langle z_{1}, z_{2}, \ldots, z_{p}\right\rangle$ such that $h\left(f_{1}, f_{2}, \ldots, f_{p}\right)=0$. 
Theorem 3. Let $f_{1}, f_{2}, \ldots, f_{p}$ be elements of the free Novikov algebra $N_{0}\left\langle x_{1}, x_{2}, \ldots, x_{n}\right\rangle$ (without identity). The elements $f_{1}, f_{2}, \ldots, f_{p}$ are Novikov dependent if and only if they are differentially algebraically dependent in $k\left\{x_{1}, x_{2}, \ldots, x_{n}\right\}$.

Proof. Suppose that the elements $f_{1}, f_{2}, \ldots, f_{p}$ of the Novikov algebra $N_{0}\left\langle x_{1}, x_{2}, \ldots, x_{n}\right\rangle$ are Novikov dependent. Let $h\left(z_{1}, z_{2}, \ldots, z_{p}\right) \in N\left\langle z_{1}, z_{2}, \ldots, z_{p}\right\rangle$ be a nonzero element such that $h\left(f_{1}, f_{2}, \ldots, f_{p}\right)=0$. Since $f_{1}, f_{2}, \ldots, f_{p}$ do not contain constants, we can assume that $h\left(z_{1}, z_{2}, \ldots, z_{p}\right)$ also does not contain a constant, i.e., $h\left(z_{1}, z_{2}, \ldots, z_{p}\right) \in N_{0}\left\langle z_{1}, z_{2}, \ldots, z_{p}\right\rangle$. Consequently, $h\left(z_{1}, z_{2}, \ldots, z_{p}\right) \in k\left\{z_{1}, z_{2}, \ldots, z_{p}\right\}$. Then $h\left(f_{1}, f_{2}, \ldots, f_{p}\right)=0$ gives that $f_{1}, f_{2}, \ldots, f_{p}$ are differentially algebraically dependent.

Suppose that $f_{1}, f_{2}, \ldots, f_{p} \in N_{0}\left\langle x_{1}, x_{2}, \ldots, x_{n}\right\rangle$ are differentially algebraically dependent. Let $g\left(z_{1}, z_{2}, \ldots, z_{p}\right) \in k\left\{z_{1}, z_{2}, \ldots, z_{p}\right\}$ be a nonzero element such that $g\left(f_{1}, f_{2}, \ldots, f_{p}\right)=$ 0 . We can write $g$ in the form

$$
g=u_{t}+u_{t+1}+\ldots+u_{s}
$$

where $\rho\left(u_{i}\right)=i, t \leq i \leq s$, and $u_{s} \neq 0$. Then

$$
g\left(f_{1}, f_{2}, \ldots, f_{p}\right)=u_{t}\left(f_{1}, f_{2}, \ldots, f_{p}\right)+u_{t+1}\left(f_{1}, f_{2}, \ldots, f_{p}\right)+\ldots+u_{s}\left(f_{1}, f_{2}, \ldots, f_{p}\right)=0 .
$$

By Lemma 3 we have

$$
\overline{g\left(f_{1}, f_{2}, \ldots, f_{p}\right)}=u_{s}\left(f_{1}, f_{2}, \ldots, f_{p}\right) \text {. }
$$

It follows that $u_{s}\left(f_{1}, f_{2}, \ldots, f_{p}\right)=0$. If $s>1$, then by differentiating $u_{s}\left(f_{1}, f_{2}, \ldots, f_{p}\right)$ $s-1$ once we get the element $w_{1}$ such that

$$
w_{1}\left(f_{1}, f_{2}, \ldots, f_{p}\right)=\left(u_{s}\left(f_{1}, f_{2}, \ldots, f_{p}\right)\right)^{(s-1)}=0, \quad \rho\left(w_{1}\right)=1 .
$$

By Proposition 1, the element $w_{1}$ is an element of the Novikov algebra $N_{0}\left\langle z_{1}, z_{2}, \ldots, z_{p}\right\rangle$. Then $f_{1}, f_{2}, \ldots, f_{p}$ are Novikov dependent. If $s<1$, then multiplying $u_{s}\left(f_{1}, f_{2}, \ldots, f_{p}\right)$ by $f_{1}^{|s|+1}$ we get an element $w_{2}$ such that

$$
w_{2}\left(f_{1}, f_{2}, \ldots, f_{p}\right)=u_{s}\left(f_{1}, f_{2}, \ldots, f_{p}\right) f_{1}^{|s|+1}=0, \quad \rho\left(w_{2}\right)=1 .
$$

Therefore, the elements $f_{1}, f_{2}, \ldots, f_{p}$ are again Novikov dependent.

Corollary 2. Novikov dependence of a finite system of elements of a free Novikov algebra over a constructive field $k$ of characteristic zero is algorithmically recognizable.

Proof. Let $f_{1}, f_{2}, \ldots, f_{p} \in N\left\langle x_{1}, x_{2}, \ldots, x_{n}\right\rangle$. We can assume that $f_{1}, f_{2}, \ldots, f_{p} \in$ $N_{0}\left\langle x_{1}, x_{2}, \ldots, x_{n}\right\rangle$ since the constants do not affect the dependence of elements. Theorems 2 and 3 complete the proof.

Corollary 3. Any $n+1$ elements of a free Novikov algebra of rank $n$ over a field of characteristic zero are Novikov dependent.

Proof. It is well known [9] that any $n+1$ elements of a differential polynomial algebra of rank $n$ are differentially algebraically dependent. 


\section{REFERENCES}

[1] Cohn PM. Free ideal rings and localization in general rings. New Mathematical Monographs. Vol 3, Cambridge: Cambridge University Press; 2006.

[2] Cox D, Little J, O'Shea D. Ideals, varieties, and algorithms. An introduction to computational algebraic geometry and commutative algebra. Fourth edition. Undergraduate Texts in Mathematics. Cham: Springer; 2015.

[3] Duisengaliyeva B, Naurazbekova A, Umirbaev U. Tame and wild automorphisms of differential polynomial algebras in two variables. Fundam. Prikl. Mat. (accepted)

[4] Duisengaliyeva B, Umirbaev U. A wild automorphism of a free Novikov algebra. Siberian Electronic Mathematical Reports, 15 (2018), 1671-1679.

[5] Dzhumadil'daev AS, Lofwall C. Trees, free right-simmetric algebras, free Novikov algebras and identities. Homology Homotopy Appl. 2002; 4: 165-190.

[6] van den Essen A. Polynomial automorphisms and the Jacobian conjecture. Progress in Mathematics. Vol. 190, Basel: Birkhauser verlag; 2000.

[7] Gel'fand IM, Dorfman IYa. Hamiltonian operators and algebraic structures related to them. Funct. Anal. Appl. 1979; 13: 248-262.

[8] Goodearl KR, Warfield Jr. RB. An introduction to noncommutative Noetherian rings. Second edition. London Mathematical Society Student Texts. Vol. 61, Cambridge: Cambridge University Press; 2004.

[9] Kolchin E. Differential Algebra and Algebraic Groups. Pure and Applied Mathematics. Vol. 54, New York-London: Academic Press; 1973.

[10] Kondratieva MV, Levin AB, Mikhalev AV, Pankratiev EV. Differential and difference dimension polynomials. Mathematics and its Applications. Vol. 461, Dordrecht: Kluwer Academic Publishers; 1999.

[11] Kondratieva MV, Zobnin AI. The membership problem for differential ideals generated by a composition of polynomials. Program. Comput. Softw. 2006; 32: 123-127.

[12] Magnus W, Karrass A, Solitar D. Combinatorial group theory. Presentations of groups in terms of generators and relations. Reprint of the 1976 second edition. Mineola (NY): Dover Publications, Inc.; 2004.

[13] Makar-Limanov L, Shestakov I. Polynomial and Poisson dependence in free Poisson algebras and free Poisson fields. J. Algebra. 2012; 349: 372-379.

[14] Makar-Limanov L, Umirbaev U. Free Poisson fields and their automorphisms. J. Algebra Appl. 2016; 15: 1650196 (13 pages).

[15] Noskov G. The cancellation problem for a ring of polynomials. (Russian) Sibirsk. Mat. Zh. 1978; 19: 1413-1414.

[16] Ritt J. Differential Algebra. New York: Dover Publications, Inc.; 1966.

[17] Shannon D, Sweedler M. Using Gröbner bases to determine algebra membership, split surjective algebra homomorphisms determine birational equivalence. Computational aspects of commutative algebra. J. Symbolic Comput. 1988; 6: 267-273.

[18] Shestakov I, Umirbaev U. Poisson brackets and two generated subalgebras of rings of polynomials. J. Amer. Math. Soc. 2004; 17: 181-196.

[19] Shirshov AI. Subalgebras of free Lie algebras. (Russian) Mat. Sbornik N.S. 1953; 33(75): 441-452.

[20] Umirbaev U. Some algorithmic questions concerning associative algebras. Algebra Logic. 1993; 32: $244-255$.

[21] Umirbaev U. Universal enveloping algebras and universal derivations of Poisson algebras. J. Algebra. 2012; 354: 77-94.

[22] Umirbaev U. Algorithmic problems for differential polynomial algebras. J. Algebra. 2016; 455: 77-92.

[23] Witt E. Die Unterringe der freien Lieschen Ringe. Math. Z. 1956; 64: 195-216. 